\title{
Early Effect of Amyloid $\beta$-Peptide on Hippocampal and Serum Metabolism in Rats Studied by an Integrated Method of NMR-Based Metabolomics and ANOVA-Simultaneous Component Analysis
}

\author{
Yao Du, ${ }^{1}$ Hong Zheng, ${ }^{1}$ Huanhuan Xia, ${ }^{1}$ Liangcai Zhao, ${ }^{1}$ Wenyi Hu, \\ Guanghui Bai, ${ }^{2}$ Zhihan Yan, ${ }^{2}$ and Hongchang Gao ${ }^{1}$ \\ ${ }^{1}$ School of Pharmaceutical Sciences, Wenzhou Medical University, Wenzhou 325035, China \\ ${ }^{2}$ Radiology Department, The Second Affiliated Hospital, Wenzhou Medical University, Wenzhou 325027, China
}

Correspondence should be addressed to Zhihan Yan; zhihanyan@hotmail.com and Hongchang Gao; gaohc27@wmu.edu.cn

Received 21 July 2016; Accepted 27 October 2016; Published 24 January 2017

Academic Editor: Mauro S. Oliveira

Copyright (C) 2017 Yao Du et al. This is an open access article distributed under the Creative Commons Attribution License, which permits unrestricted use, distribution, and reproduction in any medium, provided the original work is properly cited.

\begin{abstract}
Amyloid $\beta(\mathrm{A} \beta)$ deposition has been implicated in the pathogenesis of Alzheimer's disease. However, the early effect of $\mathrm{A} \beta$ deposition on metabolism remains unclear. In the present study, thus, we explored the metabolic changes in the hippocampus and serum during first 2 weeks of $\mathrm{A} \beta_{25-35}$ injection in rats by using an integrated method of NMR-based metabolomics and ANOVAsimultaneous component analysis (ASCA). Our results show that $\mathrm{A} \beta_{25-35}$ injection, time, and their interaction had statistically significant effects on the hippocampus and serum metabolome. Furthermore, we identified key metabolites that mainly contributed to these effects. After $\mathrm{A} \beta_{25-35}$ injection from 1 to 2 weeks, the levels of lactate, $\mathrm{N}$-acetylaspartate, creatine, and taurine were decreased in rat hippocampus, while an increase in lactate and decreases in LDL/VLDL and glucose were observed in rat serum. Therefore, we suggest that the reduction in energy and lipid metabolism as well as an increase in anaerobic glycolysis may occur at the early stage of $\mathrm{A} \beta_{25-35}$ deposition.
\end{abstract}

\section{Introduction}

Alzheimer's disease (AD) has been considered as a main cause of dementia [1]. In the world, there were 24.3 million people with dementia in 2001, and this number will be raised to 42.3 million in 2020 and 81.1 million by 2040 [2]. The two main pathological features of $\mathrm{AD}$ are amyloid plaques and neurofibrillary tangles [1]. At present, the wildly accepted pathogenesis of AD is the amyloid cascade hypothesis, which suggests that amyloid $\beta(\mathrm{A} \beta)$ deposition may trigger neuronal dysfunction and death in the brain [2]. In addition, AD has been associated with a metabolic disease accompanied by impairments in glucose utilization and energy metabolism [3]. Therefore, exploring the early impact of $A \beta$-peptide deposited in the hippocampus on metabolism will advance understanding of the onset and development of AD.
Metabolomics is a relatively new "omics" technique that attempts to profile all low-molecular weight metabolites in biomaterials and examines their changes induced by diseases. Metabolomics has been used as a promising tool to explore the metabolic mechanisms of AD in both human patients and animal models [4]. Modern analytical platforms that have been applied for metabolomic researches mainly include mass spectrometry (MS) and nuclear magnetic resonance (NMR) spectroscopy [5]. The application of MSbased metabolomics has been reported to identify biomarkers for AD diagnosis [6-8], to monitor disease progression $[9,10]$, to investigate therapeutic response $[11,12]$, and to explore metabolic mechanisms [13-15]. Compared with mass spectrometry analysis, NMR spectroscopy as a commonly used analytical platform in metabolomics possesses several advantages, such as simple sample preparation, high 
reproducibility, and fast analysis. NMR-based metabolomics has been applied in exploring metabolic changes in urine, plasma, and brain of AD animal models [16, 17]. Moreover, selecting a suitable data analysis method is also of great importance for a metabolomic approach [18]. In this study, two factors, $\mathrm{A} \beta_{25-35}$ injection and time, were included. Thus, ANOVA-simultaneous component analysis (ASCA) was selected, since it can not only include underlying multiple factors and their interaction, but also facilitate interpreting the effects of different factors [19]. ASCA model has been successfully applied to analyze metabolomic data; for example, Yde et al. [20] used ASCA to investigate the effects of dietary, time, and their interaction on plasma metabolome of pigs and identified different absorption patterns of betaine in response to different diets. ASCA was also used to examine different sources of variations, including dose, time, and dose/time interaction of bisphenol-A exposure, on serum metabolome, and found that energy and neurotransmitter metabolism were altered by bisphenol-A [21]. Recently, the effects of dairy intake, time, and their interaction were studied on the metabolic changes in urine, blood, and feces using ASCA [22], where it was reported that high dairy consumption may result in alterations in energy, protein, and gut microbial metabolism.

$\mathrm{A} \beta_{25-35}$ is a peptide that exists in brain of $\mathrm{AD}$ patients [23]. In animals, $A \beta_{25-35}$-administration can result in neurodegeneration $[24,25]$, inflammatory response $[25,26]$, disordered neurotransmitter metabolism [27], and impaired synaptic plasticity [4] as well as spatial learning dysfunction $[4,28]$. Therefore, $\mathrm{A} \beta_{25-35}$ injected into the temporal cortex or hippocampus of animals has been commonly used to develop AD animal models [29]. In this study, we constructed $\mathrm{AD}$ rat models by injecting $\mathrm{A} \beta_{25-35}$ into bilateral hippocampus and examined metabolic profiles in hippocampus and serum after 1 and 2 weeks of $\mathrm{A} \beta_{25-35}$ injection using NMRbased metabolomics. Then, ASCA model was used to (1) analyze the effects of $\mathrm{A} \beta_{25-35}$ injection, time, and their interaction on metabolic changes in hippocampus and serum of rats and (2) identify metabolites related to these effects.

\section{Materials and Methods}

2.1. Animals. Adult male Sprague-Dawley (SD) rats (body weight $=220 \pm 15 \mathrm{~g}$ ) were purchased from the SLAC Laboratory Animal Co. Ltd. (Shanghai, China) and kept in a specific pathogen-free colony at the Laboratory Animal Center of Wenzhou Medical University (Wenzhou, China). All rats were housed in metabolic cages with regulated temperature and humidity as well as a 12/12 h light-dark cycle with lights on at 8:00 a.m. and free access to standard rat chow and tap water. The present study was conducted according to the Guide for the Care and Use of Laboratory Animals and approved by the Institutional Animal Care and Use Committee of Wenzhou Medical University.

2.2. Brain Stereotaxic $A \beta_{25-35}$ Injection. Sixty SD rats were weighted and randomly divided into $\mathrm{A} \beta_{25-35}$ injected (AD) and control (Con) groups (30 rats for each group). After a
$12 \mathrm{~h}$ fasting, rats were anesthetized with chloroform $(3 \mathrm{~mL} / \mathrm{kg}$ of body weight) and then placed on the stereotaxic apparatus to inject $\mathrm{A} \beta_{25-35}$ solution into bilateral hippocampus CA1. $\mathrm{A} \beta_{25-35}$ (Sigma Aldrich, St. Louis, MO, USA) solution was prepared by dissolving in saline at $5 \mathrm{mg} / \mathrm{mL}$ and incubating at $37^{\circ} \mathrm{C}$ for 7 days. Firstly, we cleaned rats' scalp by iodine solution and then incised on the midline to expose the skull. After that, two holes were drilled on both sides of the middle line $(3.0 \mathrm{~mm}$ posterior to bregma, $2.2 \mathrm{~mm}$ lateral to sagittal suture, and $2.9 \mathrm{~mm}$ beneath the surface of brain, A/P 3.0, M/L 2.2 , and $\mathrm{D} / \mathrm{V} 2.9[30])$, followed by injecting $2 \mu \mathrm{L}$ of $\mathrm{A} \beta_{25-35}$ solution at the rate of $0.2 \mu \mathrm{L} / \mathrm{min}$. At the end of injection, the cannula was left for additional $5 \mathrm{~min}$ to allow sufficient diffusion of $\mathrm{A} \beta_{25-35}$ into the hippocampus. Rats in control group were treated with the same procedure but injected with saline.

2.3. Morris Water Maze (MWM) Test. The MWM test was performed to evaluate learning and memory ability in rats at 1 week after $\mathrm{A} \beta_{25-35}$ injection based on our previous method [31]. In brief, a circular pool with a diameter of $110 \mathrm{~cm}$ and a height of $30 \mathrm{~cm}$ was used and filled with opaque water at $22 \pm 2^{\circ} \mathrm{C}$. The escape platform with a diameter of $7 \mathrm{~cm}$ was submerged $1 \mathrm{~cm}$ below the surface of the water. During a 4 day training period ( 4 trials/day), rats were guided to reach the escape platform by the operator, if they were not able to get it within $60 \mathrm{~s}$. Then, the trained rats were subjected to a $90 \mathrm{~s}$ probe test without the escape platform. The swimming path, mean velocity, and the number of crossings over the original platform location were recorded using a computer system.

2.4. Sample Collection and Preparation. The rat was sacrificed by decapitation at 1 and 2 weeks after $\mathrm{A} \beta_{25-35}$ injection. The hippocampus was isolated immediately, frozen using liquid nitrogen, and stored at $-80^{\circ} \mathrm{C}$ until analysis. The hippocampus tissue was extracted according to our previous method [31]. In brief, the frozen brain tissue was weighed into an Eppendorf tube, and $4 \mathrm{~mL} / \mathrm{g}$ of cold methanol and $0.85 \mathrm{~mL} / \mathrm{g}$ of cold water were added into the sample tube. After homogenizing, $2 \mathrm{~mL} / \mathrm{g}$ of cold chloroform and $2 \mathrm{~mL} / \mathrm{g}$ of cold water were added into the mixture. Then, the mixture was homogenized using a vortex mixer, placed on ice for $15 \mathrm{~min}$, and centrifuged at $10,000 \times \mathrm{g}$ at $4^{\circ} \mathrm{C}$ for $15 \mathrm{~min}$. Finally, the supernatant was carefully transferred into a new Eppendorf tube, lyophilized for $24 \mathrm{~h}$, and stored at $-80^{\circ} \mathrm{C}$ until NMR analysis. The dried extract was redissolved in $500 \mu \mathrm{L}$ of $\mathrm{D}_{2} \mathrm{O}$ containing $0.50 \mathrm{mM}$ sodium trimethylsilyl propionate- $\mathrm{d} 4$ (TSP) and transferred to a $5 \mathrm{~mm}$ tube for NMR analysis.

Blood samples were collected from tail vein at 1 and 2 weeks after injection, centrifuged at $3,000 \times \mathrm{g}$ at $4^{\circ} \mathrm{C}$ for $15 \mathrm{~min}$ to obtain serum, and stored at $-80^{\circ} \mathrm{C}$ until NMR analysis. Prior to NMR analysis, $200 \mu \mathrm{L}$ of serum was thawed and diluted with $250 \mu \mathrm{L}$ of phosphate buffer $(0.2 \mathrm{mM}$ $\mathrm{Na}_{2} \mathrm{HPO}_{4} / \mathrm{NaH}_{2} \mathrm{PO}_{4}, \mathrm{pH} 7.4$ ) to minimize $\mathrm{pH}$ variations and with $50 \mu \mathrm{L}$ of $\mathrm{D}_{2} \mathrm{O}$ for field frequency locking. Subsequently, the diluted serum was centrifuged at $12,000 \times \mathrm{g}$ at $4^{\circ} \mathrm{C}$ for 
TABle 1: $P$ values of ASCA models by 10,000-permutation test.

\begin{tabular}{lccc}
\hline Samples & $\mathrm{A} \beta_{25-35}$ & Time & $\mathrm{A} \beta_{25-35} \times$ time \\
\hline Hippocampus & 0.0219 & 0.0001 & 0.0198 \\
Serum & 0.0065 & 0.0001 & 0.0001 \\
\hline
\end{tabular}

$10 \mathrm{~min}$. Then, $500 \mu \mathrm{L}$ of supernatant was transferred into a $5 \mathrm{~mm}$ NMR tube for NMR analysis.

2.5. NMR-Based Metabolomic Analysis. ${ }^{1} \mathrm{H}$ NMR spectra were recorded using a Bruker AVANCE III 600 NMR spectrometer at $37^{\circ} \mathrm{C}$. The Carr-Purcell-Meiboom-Gill (CPMG) pulse sequence was applied to reduce broad NMR signals from proteins and lipids. The main acquisition parameters were set as follows: acquisition time, $2.65 \mathrm{sec}$ per scan; data points, $64 \mathrm{~K}$; spectral width, $12,000 \mathrm{~Hz}$; relaxation delay, $2 \mathrm{sec}$.

NMR spectra were manually phase/baseline-corrected and referenced to TSP peak at $0.00 \mathrm{ppm}$ using Topspin software (v2.1 pl4, Bruker Biospin, Germany). All spectra were aligned using the "icoshift" procedure in MATLAB (R2012a, The Mathworks Inc., Natick, MA, USA) [32]. The spectral regions from 0.0 to $4.5 \mathrm{ppm}$ for hippocampal sample and from 0.0 to $4.2 \mathrm{ppm}$ for serum sample were subdivided and integrated to binning data with a size of $0.01 \mathrm{ppm}$ for further multivariate analysis. The NMR signals were assigned using the Chenomx NMR suite 7.0 (Chenomx Inc., Edmonton, Canada) and the Human Metabolome Database [33] as well as the reported data on brain tissue [31] and serum [34].

2.6. ANOVA-Simultaneous Component Analysis (ASCA). The ASCA model, which combines analysis of variance (ANOVA) and simultaneous component analysis (ACA), was used in this study. Two factors, $\mathrm{A} \beta_{25-35}$ injection (AD and Con) and time (1 and 2 weeks), were included in the ASCA model. Firstly, the data matrix $X(M \times N$, where $M$ is the number of samples and $N$ is the number of variables) was separated into matrices for $\mathrm{A} \beta_{25-35}$ injection $\left(X_{A}\right)$ and time $\left(X_{T}\right)$ and for interaction between the two factors $\left(X_{A T}\right)$ as well as a matrix with residuals $(E)$, as shown in

$$
X=1 m^{T}+X_{A}+X_{T}+X_{A T}+E,
$$

where $1 m^{T}$ is the overall means.

Then, matrices $X_{A}, X_{T}$, and $X_{A T}$ were decomposed into score matrices $T_{A}, T_{T}$, and $T_{A T}$, loading matrices $P_{A}, P_{T}$, and $P_{A T}$, respectively, and a residual matrix $E$ as given in

$$
X=1 m^{T}+P_{A} T_{A}+P_{T} P_{T} T_{T}+P_{A T} T_{A T}+E,
$$

where $1 m^{T}$ is the overall means, $P_{A}\left(T_{A}\right), P_{T}\left(T_{T}\right)$, and $P_{A T}\left(T_{A T}\right)$ are the score (loading) matrices of $\mathrm{A} \beta_{25-35}$ injection, time, and their interaction, respectively, and $E$ is the residual matrix.

All data were Pareto-scaled and analyzed by the ASCA model using ASCA toolbox (http://www.bdagroup.nl/Home .php) under MATLAB environment (R2012a, The Mathworks Inc., Natick, MA, USA). Moreover, the ASCA model was validated by a permutation test.

\section{Results}

3.1. Impaired Learning and Memory in Rats after $A \beta_{25-35}$ Injection. In order to assess the ability of spatial learning and memory of rats, the MWM test was concluded at 1 week after $\mathrm{A} \beta_{25-35}$ injection. During a 4-day training period, the escape latency in the $\mathrm{AD}$ rats was significantly longer than that in the Con rats on day 4 (Figure 1(a)), while no significant difference was observed in mean swimming velocity between them (Figure 1(b)). Figure 1(c) illustrates the swimming trajectory of the $\mathrm{AD}$ and Con rats in the probe test of the MWM test. It can be seen that the AD rats cannot easily cross over the original platform location relative to the Con group (Figure $1(\mathrm{c})$ ). Moreover, we can see that the AD rats had significantly lower percentages of total swimming length (Figure 1(d)) and time (Figure 1(e)) in the original platform area than the Con rats. Taken together, the MWM results revealed that spatial learning and memory ability was impaired in rats after $\mathrm{A} \beta_{25-35}$ injection.

\section{2. ${ }^{1}$ H NMR Metabolite Profiles of Hippocampus and Serum in} Rats. Figures 2(a) and 2(b) display typical ${ }^{1} \mathrm{H}$ NMR spectra of hippocampus samples obtained from the $\mathrm{AD}$ and Con rats, respectively. A total of 13 metabolites were identified, involving energy metabolism (Cre, creatine; Suc, succinate; Ala, alanine; Lac, lactate), neurotransmitters (Asp, aspartate; Glu, glutamate; Gln, glutamine; GABA, $\gamma$-aminobutyric acid; Gly, glycine), and membrane metabolism (Cho, choline; NAA, N-acetylaspartate) as well as osmoregulation (Myo, myoinositol; Tau, taurine). In addition, Figures 3(a) and 3(b) illustrate typical ${ }^{1} \mathrm{H}$ NMR spectra of serum samples obtained from the $\mathrm{AD}$ and Con rats, respectively, and 12 metabolites were identified, such as energy metabolism (glucose; For, formate; Cre, creatine; Ala, alanine; Lac, lactate), amino acid metabolism (His, histidine; Tyr, tyrosine; Gln, glutamine; Val, valine), and lipid metabolism (LDL/VLDL, low-density and very low-density lipoproteins) as well as glycoprotein (NAG, $\mathrm{N}$-acetylglycoprotein; OAG, O-acetylglycoprotein).

\subsection{Identification of Metabolic Changes in the Hippocampus} and Serum of Rats after $A \beta_{25-35}$ Injection Using ASCA. Table 1 lists $P$ values of ASCA models validated by 10,000permutation test including the effects of $\mathrm{A} \beta_{25-35}$ injection, time, and their interaction $\left(\mathrm{A} \beta_{25-35}\right.$ injection $\times$ time $)$. We found significant effects of $\mathrm{A} \beta_{25-35}$ injection on the metabolite profiles in both the hippocampus $(P=0.0219)$ and serum $(P=0.0065)$. In addition, significant time effects were also observed on the hippocampus $(P=0.0001)$ and serum $(P=0.0001)$ metabolome. Most interestingly, there were significant interaction effects of $A \beta_{25-35}$ injection and time on the hippocampus $(P=0.0198)$ and serum $(P=0.0001)$ 

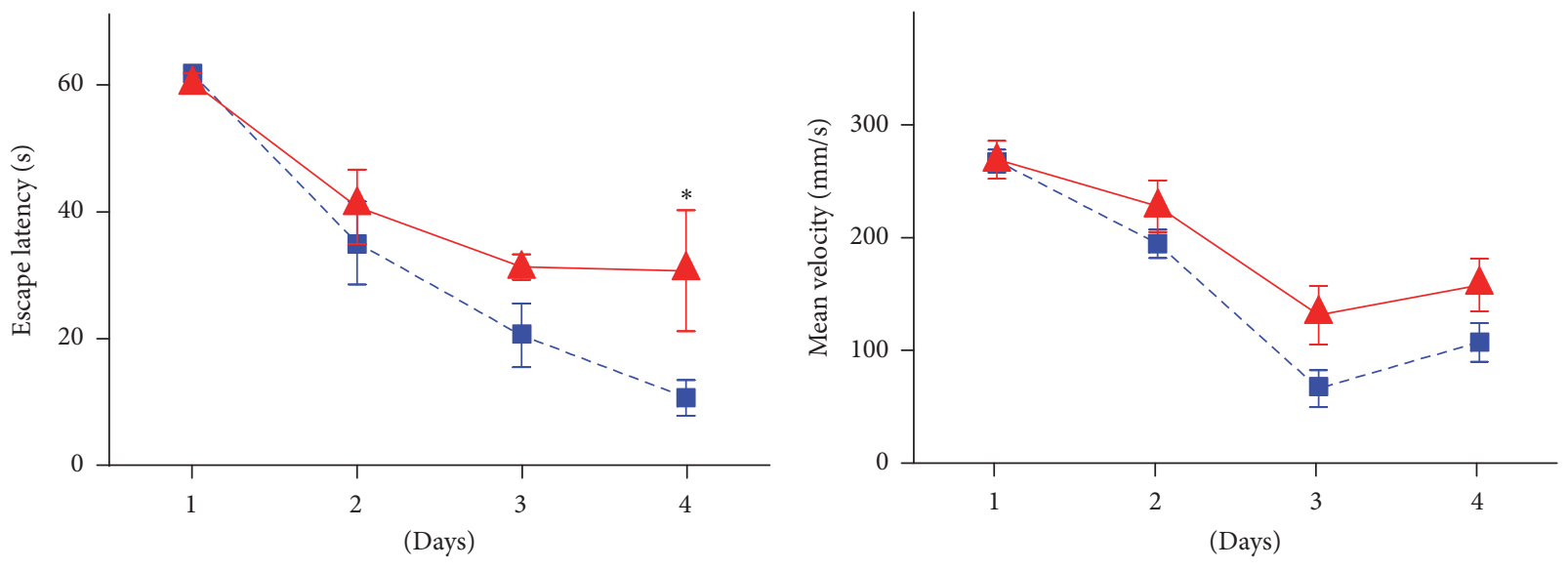

- Con
$-\mathrm{AD}$

$-1 \mathrm{Con}$
$-\mathrm{AD}$

(a)

(b)
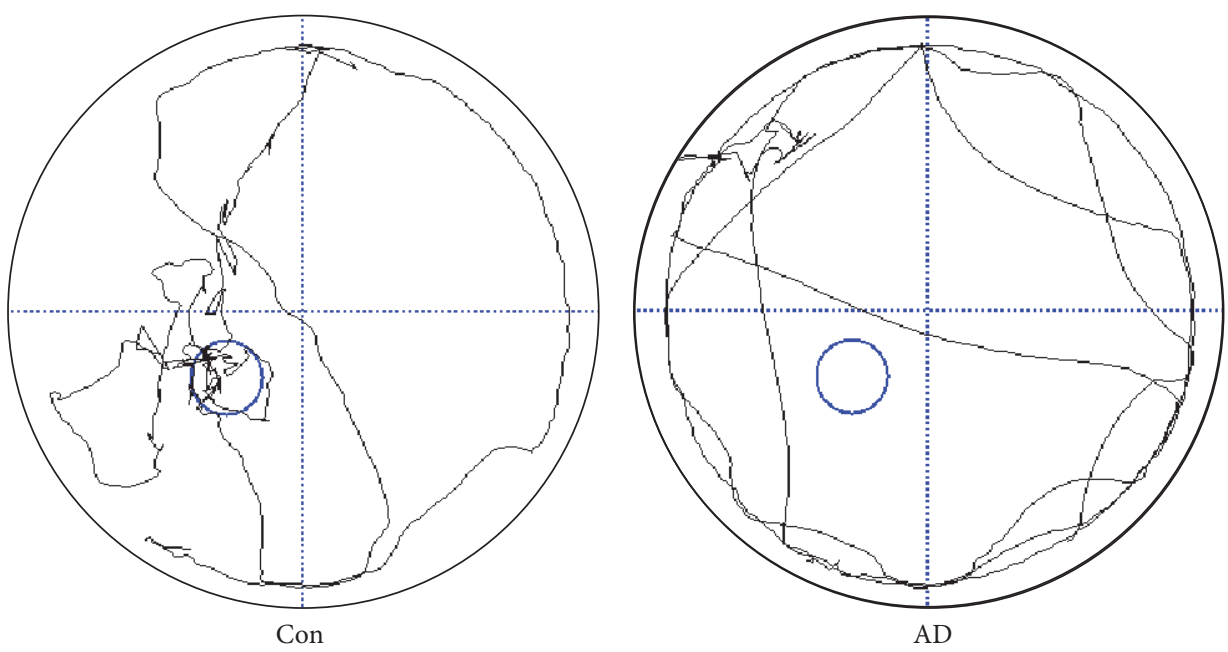

(c)

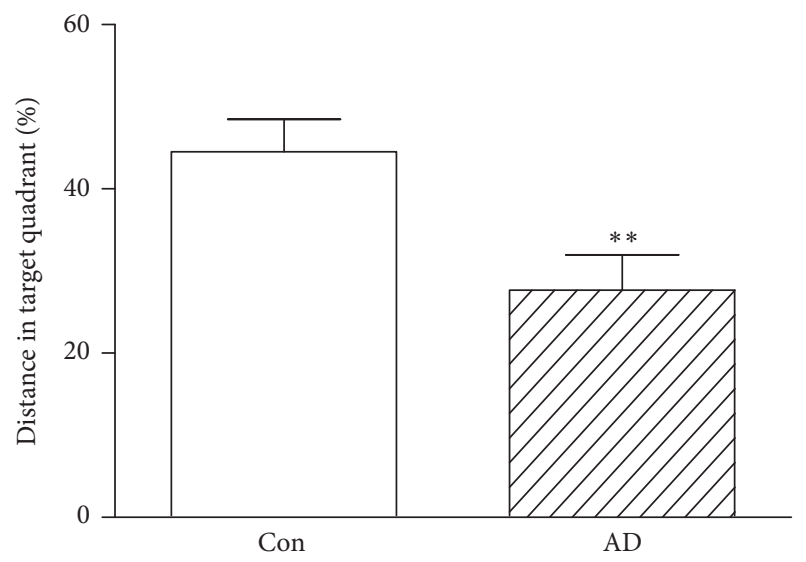

(d)

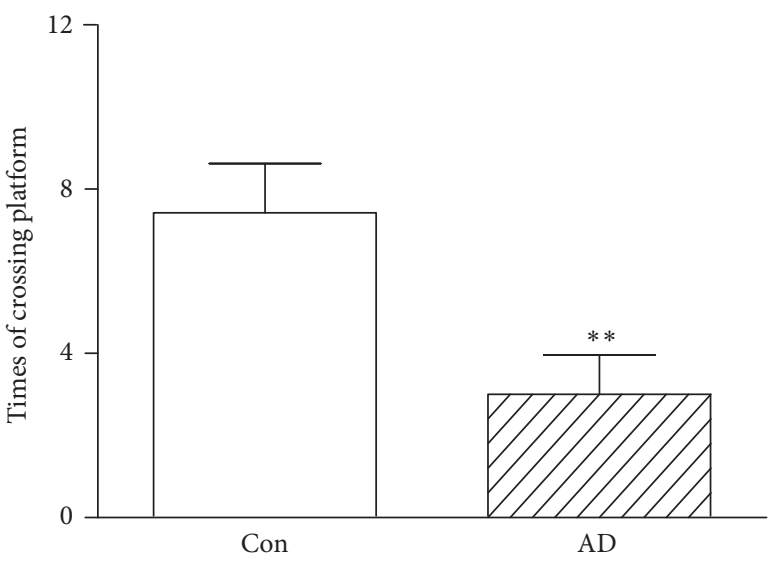

(e)

FIGURE 1: The performance of spatial learning and memory in rats after 1 week of $A \beta_{25-35}$ injection in the Morris water maze test: (a) the escape latency and (b) the mean swimming velocity during the 4-day training period, (c) the swimming path, (d) the percentage of total swimming length in the original platform area, and (e) times of crossing the original platform in the $90 \mathrm{~s}$ probe test. Significant level: ${ }^{*} P<$ $0.05 ;{ }^{* *} P<0.01$. 


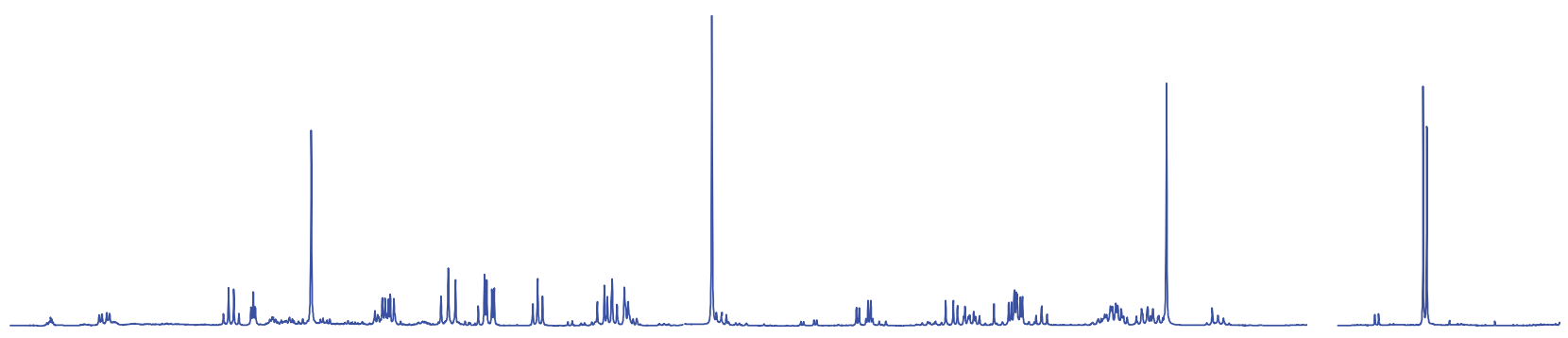

(a)

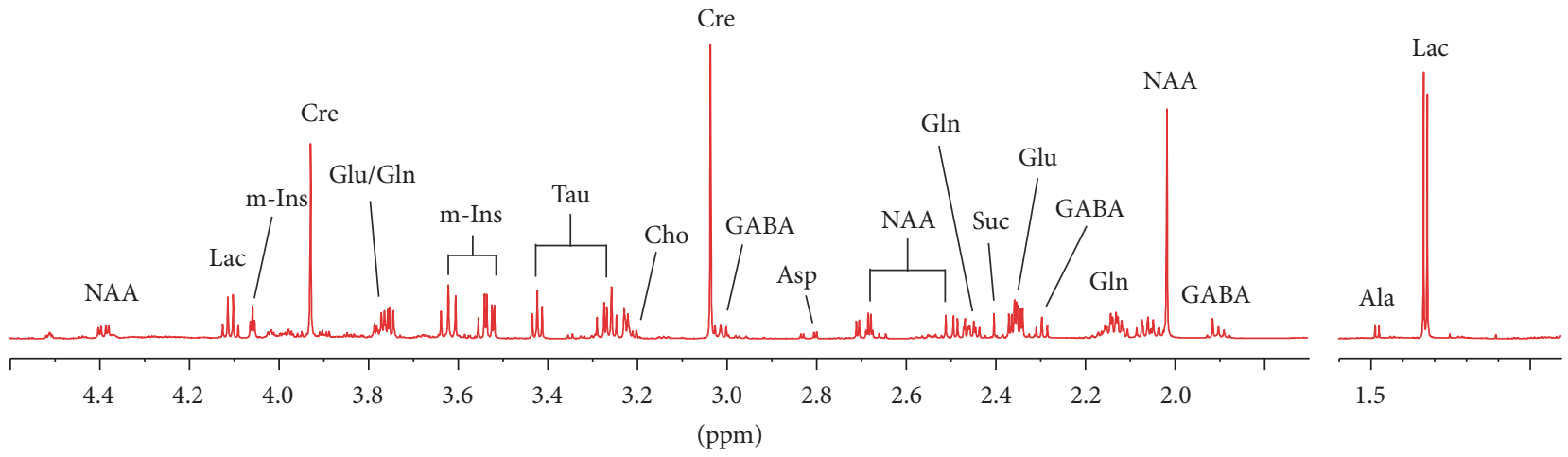

(b)

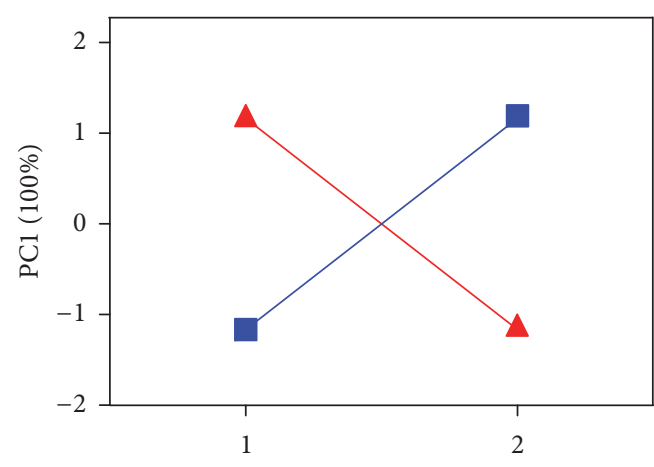

(W)

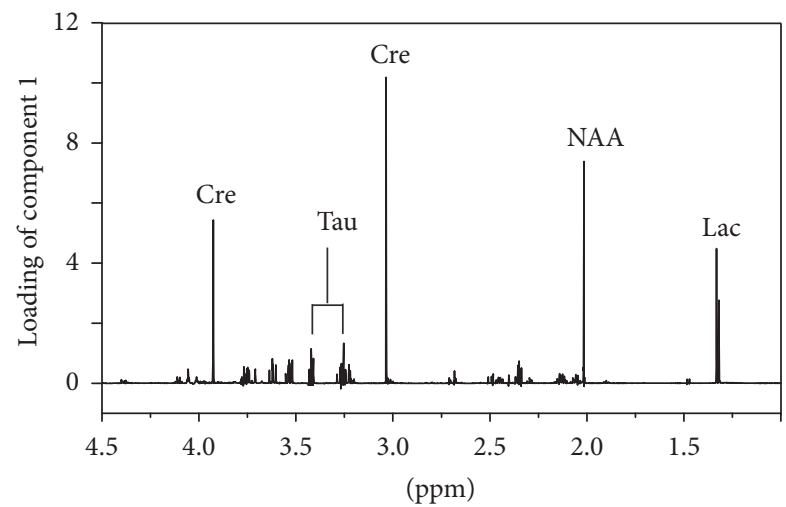

(c)

(d)

FIGURE 2: Effect of $\mathrm{A} \beta_{25-35}$ injection on hippocampal metabolism in rats analyzed by ASCA model: typical ${ }^{1} \mathrm{H}$ NMR spectra in rat hippocampus after injection of (a) saline (b) and $\mathrm{A} \beta_{25-35}$ solutions; (c) ASCA score plot of the interaction effect of $\mathrm{A} \beta_{25-35}$ injection and time as well as $(\mathrm{d})$ its corresponding loading plot. Con, the control group with saline injection; $\mathrm{AD}$, the $\mathrm{AD}$ group with $\mathrm{A} \beta_{25-35}$ injection; Ala, alanine; Lac, lactate; GABA, $\gamma$-aminobutyric acid; NAA, N-acetylaspartate; Gln, glutamine; Glu, glutamate; Suc, succinate; Asp, aspartate; Cre, creatine; Cho, choline; Tau, taurine; $\mathrm{m}$-Ins, myoinositol.

metabolome, and the corresponding ASCA score and loading plots are illustrated in Figures 2 and 3, respectively. Figure 2(d) shows that the levels of Lac, NAA, Cre, and Tau in the hippocampus were decreased after $\mathrm{A} \beta_{25-35}$ injection from 1 to 2 weeks, relative to the Con group. In the serum, we found an increase in Lac as well as a decrease in LDL/VLDL and glucose after $\mathrm{A} \beta_{25-35}$ injection from 1 to 2 weeks (Figure 3(d)).

\section{Discussion}

Amyloid $\beta$-peptide $(\mathrm{A} \beta)$ has been reported to induce oxidative stress in brain and thereby cause the onset and development of $\mathrm{AD}$ [35]. Therefore, injection of toxic $\mathrm{A} \beta$ into brain has been commonly used to construct $\mathrm{AD}$ animal model [36]. In the present study, we injected $\mathrm{A} \beta_{25-35}$ into hippocampal area CA1 of rats and expectedly found an impaired ability of spatial learning and memory after 1 week using the Morris water maze test. Exploring the early effect of $A \beta_{25-35}$ deposited in the hippocampus on metabolism will achieve a better understanding of the onset and development of $\mathrm{AD}$, but it has not been reported. Therefore, we examined the metabolic changes during first 2 weeks of $\mathrm{A} \beta_{25-35}$ injection in the hippocampus and serum of rats using an integrated method of NMR-based metabolomics and ASCA model. 


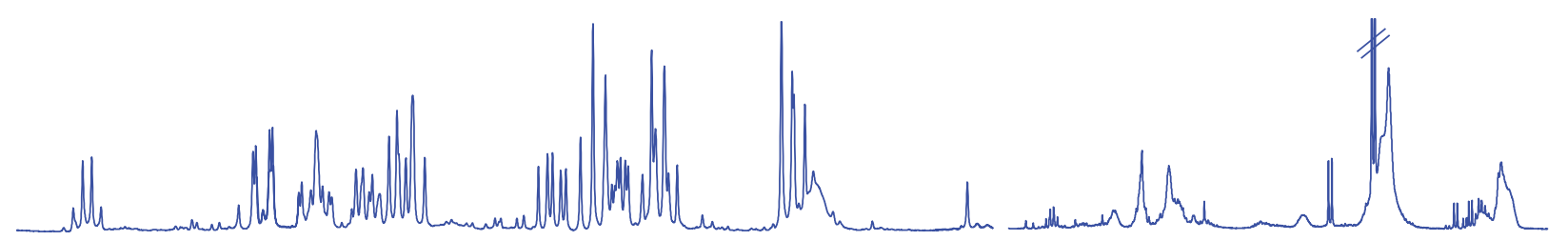

(a)
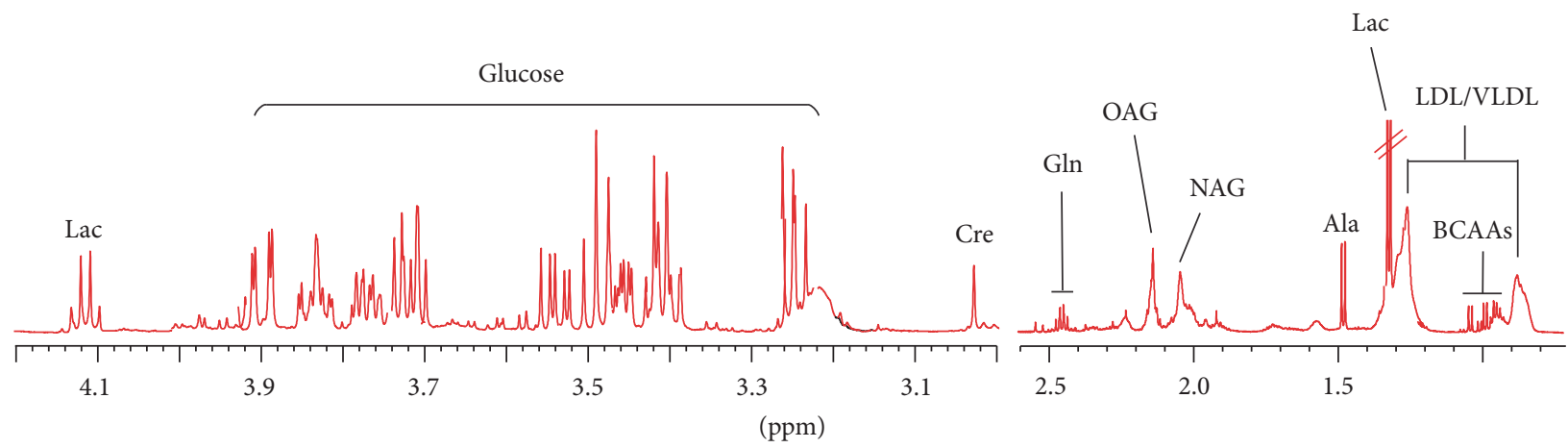

(b)

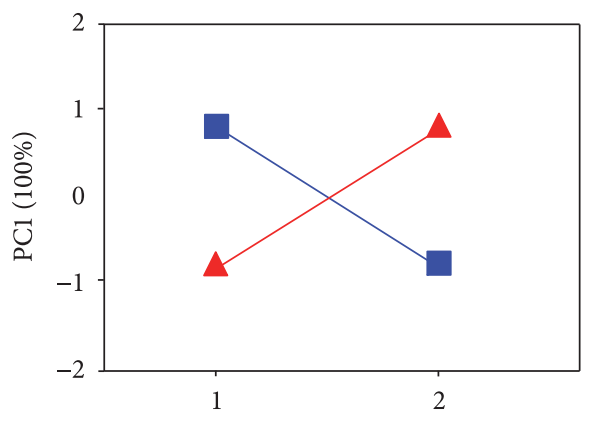

(W)

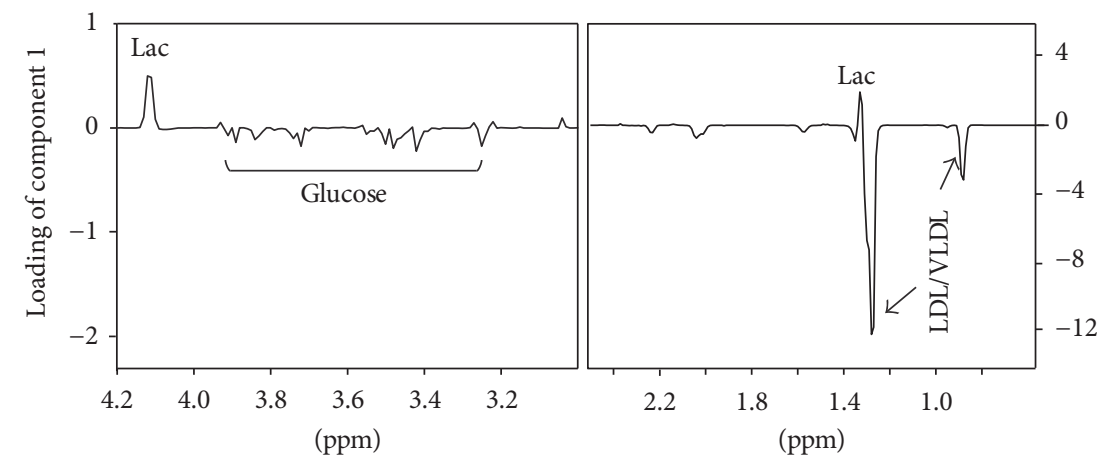

(d)

FIGURE 3: Effect of $\mathrm{A} \beta_{25-35}$ injection on serum metabolism in rats analyzed by ASCA model: typical ${ }^{1} \mathrm{H}$ NMR spectra in rat serum after injection of (a) saline (b) and $\mathrm{A} \beta_{25-35}$ solutions; (c) ASCA score plot of the interaction effect of $\mathrm{A} \beta_{25-35}$ injection and time as well as (d) its corresponding loading plot. Con, the control group with saline injection; $\mathrm{AD}$, the AD group with $\mathrm{A} \beta_{25-35}$ injection; LDL/VLDL, low-density lipoproteins and very low-density lipoproteins; Val, valine; Lac, lactate; Ala, alanine, NAG, N-acetylglycoprotein; OAG, O-acetylglycoprotein; Gln, glutamine; Cre, creatine; Tyr, tyrosine; His, histidine; For, formate.

4.1. Effect of $A \beta_{25-35}$ Injection on Hippocampal Metabolism of Rats. The brain is vulnerable to energy metabolism deficit due to its high energy consumption [37]. In general, glucose is the main substrate for brain energy metabolism [38]. However, the astrocyte-neuron lactate shuttle (ANLS) hypothesis reported that glucose is mainly metabolized to Lac in astrocytes and then transported to neurons as the primary fuel [39]. Afterwards, Lac as a neuronal energy source was also confirmed in both in vitro [40] and in vivo [41] studies. In the present study, however, we found a reduction of Lac level in rat hippocampus after $\mathrm{A} \beta_{25-35}$ injection from 1 to 2 weeks compared with that in the Con rats. In addition, Cre also plays an important role in maintaining the high energetic demand for brain development and functions via the creatine kinase/phosphocreatine system [42, 43].
Like Lac, a decreased level of Cre was also observed in rat hippocampus after $\mathrm{A} \beta_{25-35}$ injection from 1 to 2 weeks, suggesting an insufficient supply of energy in the brain of the $\mathrm{AD}$ rats. A lower Cre level was also found in the hippocampus of another AD model, TgCRND8 mice [44]. Growing evidences have supported the concept that $\mathrm{AD}$ is a metabolic disease with impaired energy metabolism [4547]. Moreover, González-Domínguez et al. [48] have also found that impaired energy metabolism is a key cause in pathogenesis of Alzheimer. Therefore, a reduction in energy metabolism in the hippocampus may be implicated in the onset and development of AD.

NAA has been regarded as a marker of neuronal density and integrity, since it is exclusively synthesized in the mitochondria of neurons $[49,50]$. We found that NAA 
level was reduced in rat hippocampus after $\mathrm{A} \beta_{25-35}$ injection relative to the Con group from 1 to 2 weeks, indicating that hippocampal neurons may be damaged during the progress of $\mathrm{AD}$ in rats. Previous findings have also shown that the level of NAA was decreased in neurodegenerative diseases, such as Alzheimer's disease and Huntington's disease [5153]. Lalande et al. [54] also found a reduction of NAA in Tg2576 mice at one month of age relative to the age-matched wild-type mice. Tau is an important indicator of astrocyte as well as a regulator of osmotic pressure $[55,56]$. Meanwhile, it also acts as an antioxidant in neuroprotection [57, 58]. In this study, compared with the Con group, a decrease in hippocampal Tau level in rats after $\mathrm{A} \beta_{25-35}$ injection from 1 to 2 weeks may reflect the reduction of astrocyte activity and antioxygenation in the hippocampus. A similar finding was also obtained in the hippocampus of TgCRND8 mice [44]. Taken together, decreased levels of NAA and Tau may indicate that astrocytes and neurons in the hippocampus were damaged during $\mathrm{AD}$ development in rats.

4.2. Effect of $A \beta_{25-35}$ Injection on Serum Metabolism of Rats. In this study, VLDL/LDL, glucose, and lactate in serum were identified as the main metabolites induced by $\mathrm{A} \beta_{25-35}$ injection using an integrated analytical method of NMRbased metabolomics and ASCA model. The initiation and acceleration of $\mathrm{AD}$ pathology have been closely associated with the downregulation of lipid metabolism $[59,60]$. In the current study, we found that the serum level of lipoprotein, VLDL/LDL, was reduced after $\mathrm{A} \beta_{25-35}$ injection from 1 to 2 weeks as compared with the Con group. Lipoprotein particles are lipid transporters in blood, so our finding may indicate a reduction of lipid metabolism in the $\mathrm{AD}$ rats. De La Monte et al. [45] found that lipid derivatives including phospholipids and sphingomyelins were significantly decreased in APP/PS1 mice. Glucose is the predominant energy substrate that can be oxidized to $\mathrm{CO}_{2}$ and $\mathrm{H}_{2} \mathrm{O}$ through tricarboxylic acid (TCA) cycle or transformed into lactate by anaerobic glycolysis. In this study, an increase in lactate level and a decrease in glucose level were found in the serum after $\mathrm{A} \beta_{25-35}$ injection from 1 to 2 weeks, indicating that anaerobic glycolysis was upregulated during AD development. Using ${ }^{1} \mathrm{H}$ NMR-based metabolomic approach, similar results were also observed in APP/PS1 [7] and senescence-accelerated mouse prone 8 (SAMP8) [46] mice. In addition, compared with the wild-type mice, De La Monte et al. [45] found reduced glucose and increased lactate in serum of the APP/PS1 mice using a MS-based metabolomic approach. Therefore, serum metabolomics analysis reveals that a decrease in lipid metabolism and an increase in anaerobic glycolysis may be associated with the development of $\mathrm{AD}$ in rats.

In conclusion, the early effect of $\mathrm{A} \beta_{25-35}$ deposition on hippocampal and serum metabolism was explored by an integrated analytical method of NMR-based metabolomics and ASCA model. Our results revealed that a series of metabolic disorders occurred at the early stage of $\mathrm{A} \beta_{25-35}$ deposition, including reduction in energy metabolism in the hippocampus as well as a decrease in lipid metabolism and an increase in anaerobic glycolysis in the serum. However, several limitations in the present study should be considered: (1) these findings should be confirmed by the use of multidose $\mathrm{A} \beta_{25-35}$ and other animal models; (2) only a few metabolites induced by $\mathrm{A} \beta_{25-35}$ injection were identified, so we suggest using a multianalytical platform for drawing a more detailed metabolic pathway; (3) measuring key proteins/enzymes in metabolic pathways will advance understanding of the metabolic mechanism underlying the onset and development of AD.

\section{Competing Interests}

The authors declare that they have no conflict of interests.

\section{Authors' Contributions}

Hongchang Gao, Liangcai Zhao, Zhihan Yan, and Hong Zheng contributed to experimental design. Yao $\mathrm{Du}$ and Huanhuan Xia contributed to animal experiment and NMR analysis. Yao Du, Hong Zheng, and Hongchang Gao contributed to data analysis, result interpretation, and writing. All authors have read, revised, and approved the final manuscript. Yao Du and Hong Zheng contributed equally to this work.

\section{Acknowledgments}

This study was supported by the National Natural Science Foundation of China (nos. 21575105 and 81501303) and Natural Science Foundation of Zhejiang Province (nos. LY14H090014, LY15H180010, and LY16H180009).

\section{References}

[1] K. Blennow, M. J. de Leon, and H. Zetterberg, "Alzheimer's disease," Lancet, vol. 368, no. 9533, pp. 387-403, 2006.

[2] C. P. Ferri, M. Prince, C. Brayne et al., "Global prevalence of dementia: a Delphi consensus study," The Lancet, vol. 366, no. 9503, pp. 2112-2117, 2005.

[3] S. M. De la Monte, “Type 3 diabetes is sporadic Alzheimer's disease: mini-review," European Neuropsychopharmacology, vol. 24, no. 12, pp. 1954-1960, 2014.

[4] E. Trushina and M. M. Mielke, "Recent advances in the application of metabolomics to Alzheimer's Disease," Biochimica et Biophysica Acta - Molecular Basis of Disease, vol. 1842, no. 8, pp. 1232-1239, 2014.

[5] A. Zhang, H. Sun, P. Wang, Y. Han, and X. Wang, "Modern analytical techniques in metabolomics analysis," Analyst, vol. 137, no. 2, pp. 293-300, 2012.

[6] R. González-Domínguez, T. García-Barrera, and J. L. GómezAriza, "Metabolomic study of lipids in serum for biomarker discovery in Alzheimer's disease using direct infusion mass spectrometry," Journal of Pharmaceutical \& Biomedical Analysis, vol. 98, pp. 321-326, 2014.

[7] R. Casanova, S. Varma, B. Simpson et al., "Blood metabolite markers of preclinical Alzheimer's disease in two longitudinally followed cohorts of older individuals," Alzheimer's \& Dementia, vol. 12, no. 7, pp. 815-822, 2016.

[8] K. Inoue, H. Tsuchiya, T. Takayama et al., "Blood-based diagnosis of Alzheimer's disease using fingerprinting metabolomics 
based on hydrophilic interaction liquid chromatography with mass spectrometry and multivariate statistical analysis," Journal of Chromatography B, vol. 974, pp. 24-34, 2015.

[9] R. González-Domínguez, A. García, T. García-Barrera, C. Barbas, and J. L. Gómez-Ariza, "Metabolomic profiling of serum in the progression of Alzheimer's disease by capillary electrophoresis-mass spectrometry," Electrophoresis, vol. 35, no. 23, pp. 3321-3330, 2014.

[10] G. Paglia, O. Miedico, A. Cristofano et al., "Distinctive pattern of serum elements during the progression of Alzheimer's disease," Scientific Reports, vol. 6, article 22769, 2016.

[11] H. Chu, A. Zhang, Y. Han et al., "Metabolomics approach to explore the effects of Kai-Xin-San on Alzheimer's disease using UPLC/ESI-Q-TOF mass spectrometry," Journal of Chromatography B: Analytical Technologies in the Biomedical and Life Sciences, vol. 1015-1016, pp. 50-61, 2016.

[12] N. Li, Y. Liu, W. Li et al., "A uplc/ms-based metabolomics investigation of the protective effect of ginsenosides rg1 and rg2 in mice with alzheimer's disease," Journal of Ginseng Research, vol. 40, no. 1, pp. 9-17, 2016.

[13] L. Whiley, A. Sen, J. Heaton et al., "Evidence of altered phosphatidylcholine metabolism in Alzheimer's disease," Neurobiology of Aging, vol. 35, no. 2, pp. 271-278, 2014.

[14] G. Paglia, M. Stocchero, S. Cacciatore et al., "Unbiased metabolomic investigation of Alzheimer's disease brain points to dysregulation of mitochondrial aspartate metabolism," Journal of Proteome Research, vol. 15, no. 2, pp. 608-618, 2016.

[15] B. Ansoleaga, M. Jové, A. Schlüter et al., "Deregulation of purine metabolism in Alzheimer's disease," Neurobiology of Aging, vol. 36, no. 1, pp. 68-80, 2015.

[16] S. F. Graham, C. Holscher, P. McClean, C. T. Elliott, and B. D. Green, " ${ }^{1} \mathrm{H}$ NMR metabolomics investigation of an Alzheimer's disease (AD) mouse model pinpoints important biochemical disturbances in brain and plasma," Metabolomics, vol. 9, no. 5, pp. 974-983, 2013.

[17] K. Fukuhara, A. Ohno, Y. Ota et al., "NMR-based metabolomics of urine in a mouse model of Alzheimer's disease: identification of oxidative stress biomarkers," Journal of Clinical Biochemistry \& Nutrition, vol. 52, no. 2, pp. 133-138, 2013.

[18] A. Alonso, S. Marsal, and A. Juliẽ, "Analytical methods in untargeted metabolomics: state of the art in 2015," Frontiers in Bioengineering \& Biotechnology, vol. 3, p. 23, 2015.

[19] A. K. Smilde, J. J. Jansen, H. C. Hoefsloot, R. A. Lamers, J. van der Greef, and M. E. Timmerman, "ANOVA-simultaneous component analysis (ASCA): a new tool for analyzing designed metabolomics data," Bioinformatics, vol. 21, no. 13, pp. 30433048, 2005.

[20] C. C. Yde, J. J. Jansen, P. K. Theil, H. C. Bertram, and K. E. B. Knudsen, "Different metabolic and absorption patterns of betaine in response to dietary intake of whole-wheat grain, wheat aleurone or rye aleurone in catheterized pigs," European Food Research and Technology, vol. 235, no. 5, pp. 939-949, 2012.

[21] M. Tremblay-Franco, N. J. Cabaton, C. Canlet et al., "Dynamic metabolic disruption in rats perinatally exposed to low doses of bisphenol-A," PLoS ONE, vol. 10, no. 10, Article ID e0141698, 2015.

[22] H. Zheng, J. K. Lorenzen, A. Astrup et al., "Metabolic effects of a 24-week energy-restricted intervention combined with low or high dairy intake in overweight women: an NMR-based metabolomics investigation," Nutrients, vol. 8, no. 3, p. 108, 2016.
[23] T. Kubo, S. Nishimura, Y. Kumagae, and I. Kaneko, "In vivo conversion of racemized $\beta$-amyloid ( $\left.\left[\mathrm{D}-\mathrm{Ser}^{26}\right] \mathrm{A} \beta 1-40\right)$ to truncated and toxic fragments ([D-Ser $\left.\left.{ }^{26}\right] \mathrm{A} \beta 25-35 / 40\right)$ and fragment presence in the brains of Alzheimer's patients," Journal of Neuroscience Research, vol. 70, no. 3, pp. 474-483, 2002.

[24] D. K. Rush, S. Aschmies, and M. C. Merriman, "Intracerebral/ sB-amyloid(25-35) produces tissue damage: is it neurotoxic?" Neurobiology of Aging, vol. 13, no. 5, pp. 591-594, 1992.

[25] A. Diaz, D. Limon, R. Chávez, E. Zenteno, and J. Guevara, "A $\beta_{25--35}$ injection into the temporal cortex induces chronic inflammation that contributes to neurodegeneration and spatial memory impairment in rats," Journal of Alzheimer's Disease, vol. 30, no. 3, pp. 505-522, 2012.

[26] S. Rosales-Corral, D.-X. Tan, R. J. Reiter, M. Valdivia-Velázquez, J. P. Acosta-Martínez, and G. G. Ortiz, "Kinetics of the neuroinflammation-oxidative stress correlation in rat brain following the injection of fibrillar amyloid- $\beta$ onto the hippocampus in vivo," Journal of Neuroimmunology, vol. 150, no. 1-2, pp. 20-28, 2004.

[27] S. A. Litvinova, P. M. Klodt, V. S. Kudrin, V. B. Narkevich, and T. A. Voronina, "The behavior and neurotransmitter contents in brain structures of rats with Alzheimer's disease modeled by administration of A $\beta 25-35$, Neurochemical Journal, vol. 9, no. 1, pp. 39-46, 2015.

[28] A. Díaz, L. De Jesús, L. Mendieta et al., “The amyloid- $\beta_{25--35}$ injection into the CA1 region of the neonatal rat hippocampus impairs the long-term memory because of an increase of nitric oxide," Neuroscience Letters, vol. 468, no. 2, pp. 151-155, 2010.

[29] A. Nitta, A. Itoh, T. Hasegawa, and T. Nabeshima, “ $\beta$-amyloid protein-induced Alzheimer's disease animal model," Neuroscience Letters, vol. 170, no. 1, pp. 63-66, 1994.

[30] G. Paxinos and C. Watson, The Rat Brain in Stereotaxic Coordinates, 1989.

[31] H. Zheng, L. Zhao, H. Xia et al., "NMR-based metabolomics reveal a recovery from metabolic changes in the striatum of 6OHDA-induced rats treated with basic fibroblast growth factor," Molecular Neurobiology, vol. 53, no. 10, pp. 6690-6697, 2016.

[32] F. Savorani, G. Tomasi, and S. B. Engelsen, "icoshift: a versatile tool for the rapid alignment of 1D NMR spectra," Journal of Magnetic Resonance, vol. 202, no. 2, pp. 190-202, 2010.

[33] D. S. Wishart, T. Jewison, A. C. Guo et al., "HMDB 3.0- the human metabolome database in 2013," Nucleic Acids Research, vol. 41, no. 1, pp. D801-D807, 2013.

[34] N. Psychogios, D. D. Hau, J. Peng et al., "The human serum metabolome," PLoS ONE, vol. 6, no. 2, Article ID e16957, 2011.

[35] D. A. Butterfield, J. Drake, C. Pocernich, and A. Castegna, "Evidence of oxidative damage in Alzheimer's disease brain: central role for amyloid $\beta$-peptide," Trends in Molecular Medicine, vol. 7, no. 12, pp. 548-554, 2001.

[36] M. Yamada, T. Chiba, J. Sasabe et al., "Implanted cannulamediated repetitive administration of $\mathrm{A} \beta 25-35$ into the mouse cerebral ventricle effectively impairs spatial working memory," Behavioural Brain Research, vol. 164, no. 2, pp. 139-146, 2005.

[37] Z. Chen and C. Zhong, "Decoding Alzheimer's disease from perturbed cerebral glucose metabolism: implications for diagnostic and therapeutic strategies," Progress in Neurobiology, vol. 108, pp. 21-43, 2013.

[38] H. Bachelard, "Brain energy metabolism," Trends in Neurosciences, vol. 1, no. 3, p. XIV, 1978.

[39] L. Pellerin and P. J. Magistretti, "Glutamate uptake into astrocytes stimulates aerobic glycolysis: a mechanism coupling neuronal activity to glucose utilization," Proceedings of the National 
Academy of Sciences of the United States of America, vol. 91, no. 22, pp. 10625-10629, 1994.

[40] A.-K. Bouzier-Sore, P. Voisin, P. Canioni, P. J. Magistretti, and L. Pellerin, "Lactate is a preferential oxidative energy substrate over glucose for neurons in culture," Journal of Cerebral Blood Flow \& Metabolism, vol. 23, no. 11, pp. 1298-1306, 2003.

[41] M. T. Wyss, R. Jolivet, A. Buck, P. J. Magistretti, and B. Weber, "In vivo evidence for lactate as a neuronal energy source," Journal of Neuroscience, vol. 31, no. 20, pp. 7477-7485, 2011.

[42] M. Wyss and R. Kaddurah-Daouk, "Creatine and creatinine metabolism," Physiological Reviews, vol. 80, no. 3, pp. 1107-1213, 2000.

[43] R. H. Andres, A. D. Ducray, U. Schlattner, T. Wallimann, and H. R. Widmer, "Functions and effects of creatine in the central nervous system," Brain Research Bulletin, vol. 76, no. 4, pp. 329343, 2008.

[44] R. M. Salek, J. Xia, A. Innes et al., "A metabolomic study of the CRND8 transgenic mouse model of Alzheimer's disease," Neurochemistry International, vol. 56, no. 8, pp. 937-947, 2010.

[45] S. M. De La Monte, E. Re, L. Longato, and M. Tong, "Dysfunctional pro-ceramide, ER stress, and insulin/IGF signaling networks with progression of Alzheimer's disease," Journal of Alzheimer's Disease, vol. 30, supplement 2, pp. S217-S229, 2012.

[46] S. M. De La Monte, "Therapeutic targets of brain insulin resistance in sporadic Alzheimer's disease," Frontiers in Bioscience (Elite Edition), vol. 4, pp. 1582-1605, 2012.

[47] S. M. De La Monte, L. Longato, M. Tong, and J. R. Wands, "Insulin resistance and neurodegeneration: roles of obesity, type 2 diabetes mellitus and non-alcoholic steatohepatitis," Current Opinion in Investigational Drugs, vol. 10, no. 10, pp. 1049-1060, 2009.

[48] R. González-Domínguez, T. García-Barrera, J. Vitorica, and J. L. Gómez-Ariza, "Deciphering metabolic abnormalities associated with Alzheimer's disease in the APP/PS1 mouse model using integrated metabolomic approaches," Biochimie, vol. 110, pp. 119-128, 2015.

[49] K. Liu, X.-J. Ye, W.-Y. Hu et al., "Neurochemical changes in the rat occipital cortex and hippocampus after repetitive and profound hypoglycemia during the neonatal period: an ex vivo ${ }^{1} \mathrm{H}$ Magnetic Resonance Spectroscopy Study," Molecular Neurobiology, vol. 48, no. 3, pp. 729-736, 2013.

[50] X. Zhang, H. Liu, J. Wu, X. Zhang, M. Liu, and Y. Wang, "Metabonomic alterations in hippocampus, temporal and prefrontal cortex with age in rats," Neurochemistry International, vol. 54, no. 8, pp. 481-487, 2009.

[51] I. Barba, R. Fernandez-Montesinos, D. Garcia-Dorado, and D. Pozo, "Alzheimer's disease beyond the genomic era: nuclear magnetic resonance (NMR) spectroscopy-based metabolomics," Journal of Cellular and Molecular Medicine, vol. 12, no. 5A, pp. 1477-1485, 2008.

[52] M. Marjanska, G. L. Curran, T. M. Wengenack et al., "Monitoring disease progression in transgenic mouse models of Alzheimer's disease with proton magnetic resonance spectroscopy," Proceedings of the National Academy of Sciences of the United States of America, vol. 102, no. 33, pp. 11906-11910, 2005.

[53] D. S. Dunlop, D. M. Mc Hale, and A. Lajtha, "Decreased brain $\mathrm{N}$-acetylaspartate in Huntington's disease," Brain Research, vol. 580, no. 1-2, pp. 44-48, 1992.

[54] J. Lalande, H. Halley, S. Balayssac et al., " ${ }^{1}$ H NMR metabolomic signatures in five brain regions of the A $\beta$ PPswe Tg2576 mouse model of Alzheimer's disease at four ages," Journal of Alzheimers Disease, vol. 39, no. 1, pp. 121-143, 2014.
[55] H.-Y. Liu, W.-Y. Gao, W. Wen, and Y.-M. Zhang, "Taurine modulates calcium influx through L-type voltage-gated calcium channels in isolated cochlear outer hair cells in guinea pigs," Neuroscience Letters, vol. 399, no. 1-2, pp. 23-26, 2006.

[56] Y. Xiang, H. Gao, H. Zhu, N. Sun, Y. Ma, and H. Lei, "Neurochemical changes in brain induced by chronic morphine treatment: NMR studies in thalamus and somatosensory cortex of rats," Neurochemical Research, vol. 31, no. 10, pp. 1255-1261, 2006.

[57] M. A. S. Di Leo, S. A. Santini, N. Gentiloni Silveri, B. Giardina, F. Franconi, and G. Ghirlanda, "Long-term taurine supplementation reduces mortality rate in streptozotocin-induced diabetic rats," Amino Acids, vol. 27, no. 2, pp. 187-191, 2004.

[58] J. Zhou, Y. Li, G. Yan et al., "Protective role of taurine against morphine-induced neurotoxicity in C6 cells via inhibition of oxidative stress," Neurotoxicity Research, vol. 20, no. 4, pp. 334342, 2011.

[59] M. M. Mielke and C. G. Lyketsos, "Lipids and the pathogenesis of Alzheimer's disease: is there a link?" International Review of Psychiatry, vol. 18, no. 2, pp. 173-186, 2006.

[60] J. K. Yao, T. M. Wengenack, G. L. Curran, and J. F. Poduslo, "Reduced membrane lipids in the cortex of alzheimer's disease transgenic mice," Neurochemical Research, vol. 34, no. 1, pp. 102 108, 2009. 

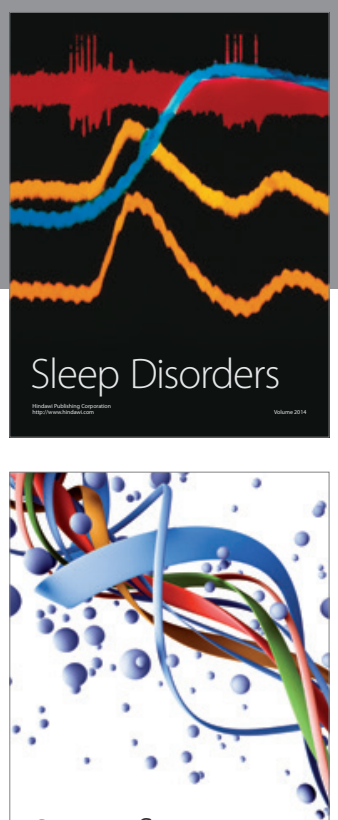

Scientifica
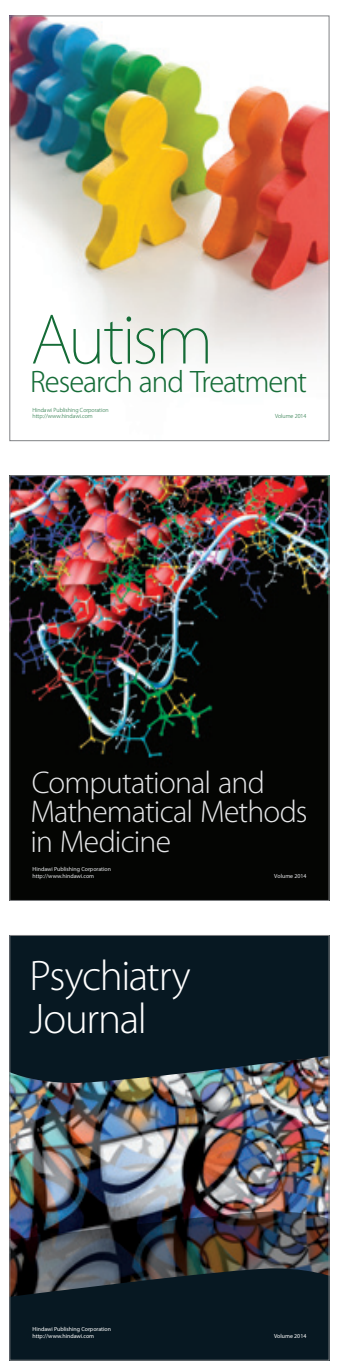
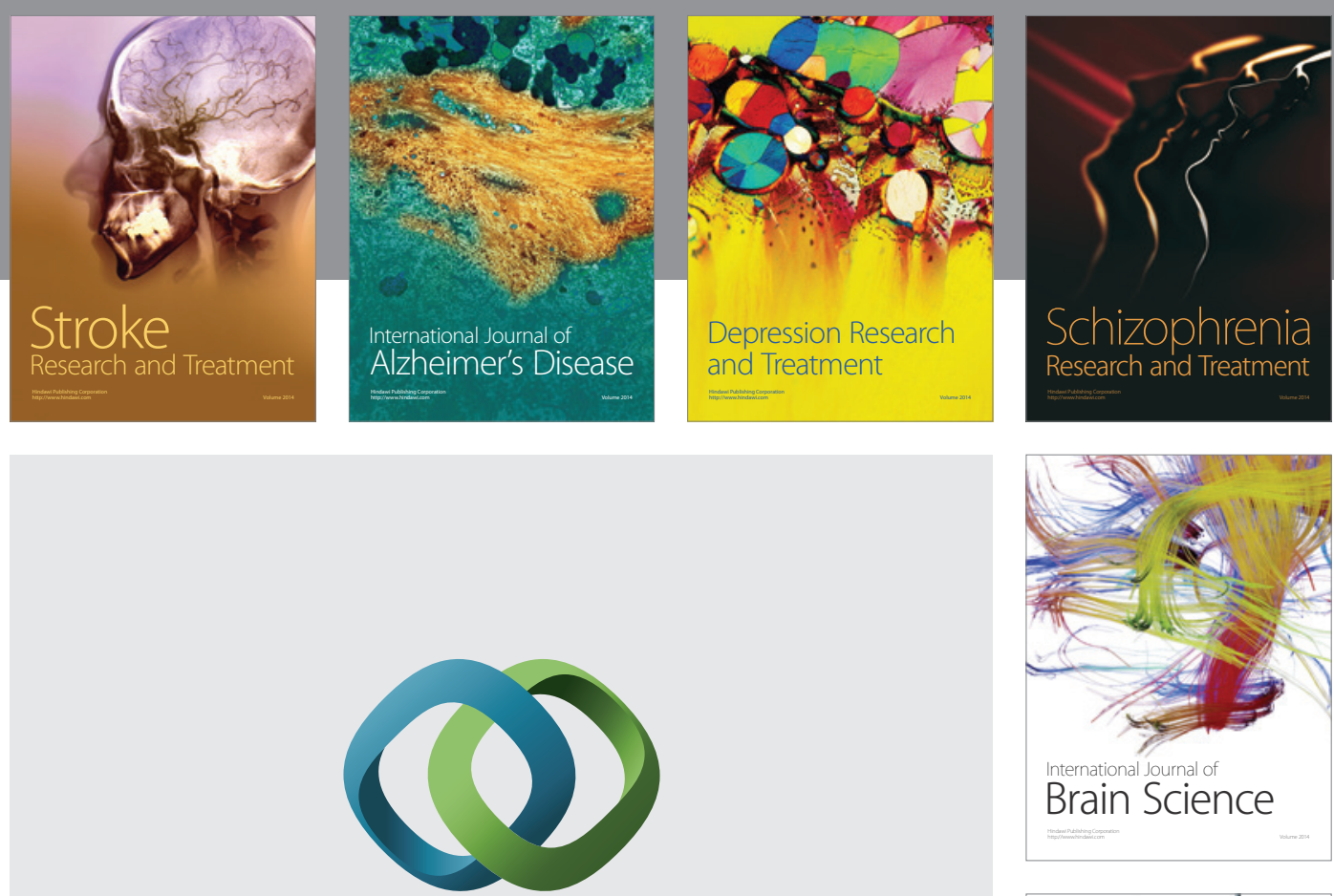

\section{Hindawi}

Submit your manuscripts at

https://www.hindawi.com
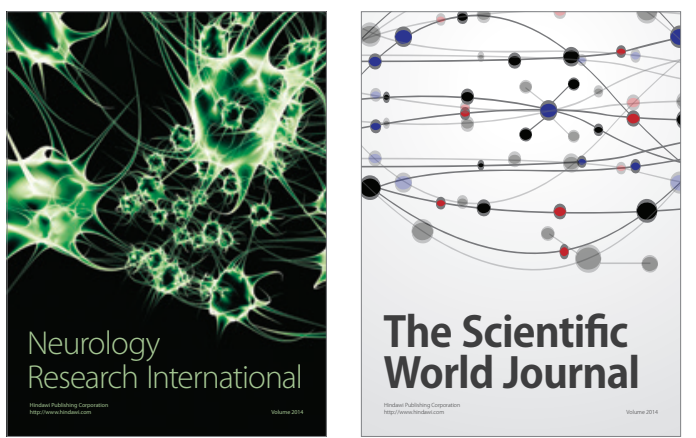

The Scientific World Journal

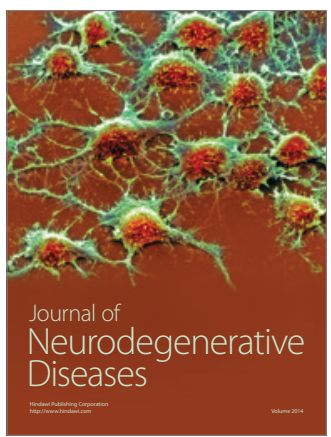

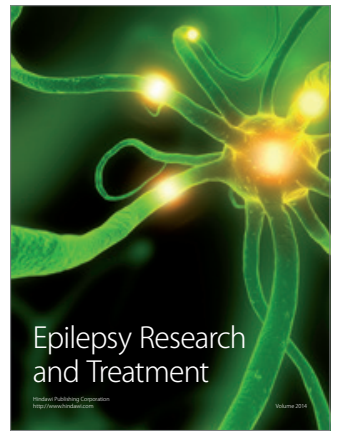

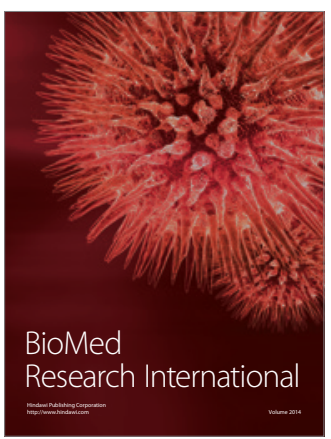

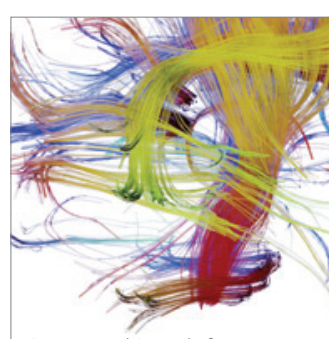

Brain Science

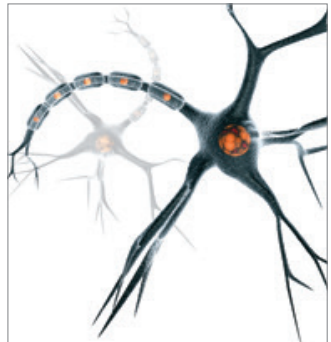

Neural Plasticity
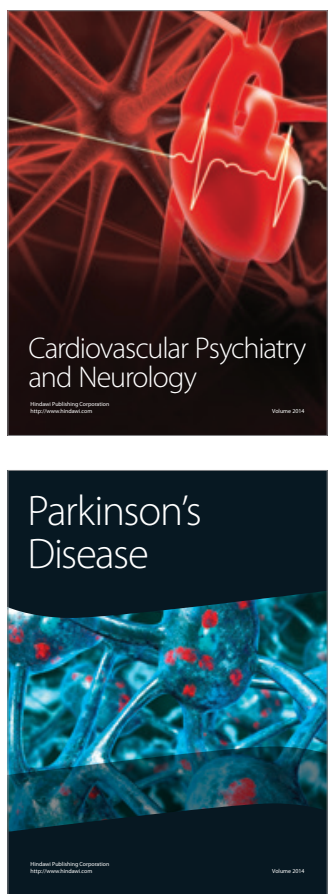